Pacific Journal of Mathematics

A DUALITY THEOREM FOR EXTENSIONS OF INDUCED 


\title{
A DUALITY THEOREM FOR EXTENSIONS OF INDUCED HIGHEST WEIGHT MODULES
}

\author{
David H. Collingwood and Brad Shelton
}

\begin{abstract}
We begin by recalling that homogeneous differential operators between smooth vector bundles over a real flag manifold correspond to the intertwining maps between algebraically induced highest weight modules. Within this framework we prove a duality theorem for extensions of induced highest weight modules. In particular, this leads to a duality theory for the nilpotent cohomology of any generalized Verma module.
\end{abstract}

1. Introduction. In this short note we recall (and prove) a folklore result which appeared in Boe's 1982 Yale thesis (without proof) and was attributed to G. Zuckerman. We apply the result to representations of real groups and to the theory of highest weight modules. In particular we obtain a duality theorem for extensions between parabolically induced highest weight modules, cf. Theorem 1.1 below. Nontrivial applications are discussed in $\S \S 4$ and 5.

Fix a pair $(\mathfrak{g}, \mathfrak{p}), \mathfrak{g}$ a complex semisimple Lie algebra and $\mathfrak{p}$ a parabolic subalgebra. There exists a connected real semisimple matrix group $G$ with a closed parabolic subgroup $P$ so that $\mathfrak{g}$ and $\mathfrak{p}$ are the complexified Lie algebras of $G$ and $P$ respectively. Let $\mathfrak{p}=\mathfrak{l} \oplus \mathfrak{n}$ be a Levi decomposition of $\mathfrak{p}$ and $\mathfrak{h} \subseteq \mathfrak{l}$ a Cartan subalgebra of both $\mathfrak{l}$, the reductive part of $\mathfrak{p}$, and of $\mathfrak{g}$.

Recall the category $\mathscr{O}_{\mathfrak{p}}$ of finitely generated $\mathfrak{g}$-modules which are $\mathfrak{l}$-semisimple and $\mathfrak{p}$-locally finite. Denote by $\mathscr{A}_{\mathfrak{p}}$ the category of finite dimensional $\mathfrak{p}$-modules which are $\mathfrak{l}$-semisimple. Define a functor $U_{\mathfrak{p}}: \mathscr{A}_{\mathfrak{p}} \rightarrow \mathscr{O}_{\mathfrak{p}}$ by

$$
U_{\mathfrak{p}}(E)=U(\mathfrak{g}) \otimes_{U(\mathfrak{p})} E .
$$

Here $U(\mathfrak{a})$ denotes the enveloping algebra of a Lie algebra $\mathfrak{a}$. For any finite dimensional $\mathfrak{p}$-module (or $P$-module) $E$, let $E^{*}$ denote the contragradient module. Our main result is then

Theorem 1.1. For any two $\mathfrak{p}$-modules $E$ and $F$ in $\mathscr{A}_{\mathfrak{p}}$ and any $k \geq 0$,

$$
\operatorname{dim}_{\mathbb{C}} \operatorname{Ext}_{\mathscr{O}_{\mathfrak{p}}}^{k}\left(U_{\mathfrak{p}}(E), U_{\mathfrak{p}}(F)\right)=\operatorname{dim}_{\mathbb{C}} \operatorname{Ext}_{\mathscr{O}_{\mathfrak{p}}}^{k}\left(U_{\mathfrak{p}}\left(F^{*}\right), U_{\mathfrak{p}}\left(E^{*}\right)\right) .
$$


Using standard homological algebra we can restate a special case of Theorem 1.1 as a duality for the nilpotent cohomology of certain standard induced modules, the generalized Verma modules.

COROLlary 1.2. Let $E$ and $F$ be irreducible finite dimensional $\mathfrak{l}$ modules and extend these to be $\mathfrak{p}$-modules by letting $\mathfrak{n}$ act trivially. Then for all $k \geq 0$,

$$
\operatorname{dim}_{\mathbb{C}} \operatorname{Hom}_{\mathfrak{l}}\left(E, H^{k}\left(\mathfrak{n}, U_{\mathfrak{p}}(F)\right)\right)=\operatorname{dim}_{\mathbb{C}} \operatorname{Hom}_{\mathfrak{l}}\left(F^{*}, H^{k}\left(\mathfrak{n}, U_{\mathfrak{p}}\left(E^{*}\right)\right)\right) .
$$

In category $\mathscr{O}_{\mathfrak{p}}$ there is no natural contravariant duality functor which carries induced modules into induced modules. Thus, in order to prove Theorem 1.1 we transfer the problem into the smooth vector bundle category where there is a natural duality, the adjoint. The existence of this duality is directly related to the existence of Haar measure which has no counterpart in category $\mathscr{O}_{\mathfrak{p}}$.

2. Homogeneous differential operators. In this section we establish the folklore result mentioned above, relating homogeneous differential operators between vector bundles to $\mathfrak{g}$-homomorphisms between induced modules. This lemma seems to be known to experts; see for example the introduction to Lepowsky's paper [L] or $\S 2$ of EastwoodRice [ER]. A special case of the lemma appears in a paper of Jakobsen, [J]. (Jakobsen and Eastwood-Rice work in the holomorphic category. In contrast, we will work in the smooth category so as to allow applications to real noncomplex Lie groups.) However, we know of no general reference with proof in the literature. The proof below is a synthesis of conversations with B. Boe, H. Schlicktkrull and G. Zuckerman. Because we have in mind future applications to the theory of Harish-Chandra modules for real reductive Lie groups, we work in a slightly more general setting than necessary for the proof of Theorem 1.1 .

Recall the Iwasawa decomposition $G=K A_{m} N_{m}, K$ a maximal compact subgroup of $G$ and $\theta$ a corresponding Cartan involution. Let $P_{m}=M_{m} A_{m} N_{m}$ be a compatible minimal parabolic subgroup. We may assume $P_{m} \subseteq P$. Let $P$ have Levi decomposition $P=L N$ and recall that $P$ is not, in general, connected. Let $P^{0}$ denote the connected component of the identity in $P$. Fix a closed subgroup $P^{\prime}$ with $P^{0} \subseteq P^{\prime} \subseteq P$ and put $L^{\prime}=L \cap P^{\prime}$. Since $G$ is linear, there is a finite $\mathbb{Z}_{2}$-group $S \subset K$ with $P^{\prime}=S P^{0}$, cf. [KZ]. Also, since $G$ is linear and $K$ is compact, $G / P_{m}^{0} \cong K / M_{m}^{0}$ is a compact connected real 
analytic manifold. Put $X=G / P^{\prime}$. Since $P_{m}^{0} \subseteq P^{\prime}, X$ is a compact manifold.

Let $(\sigma, E)$ be a finite dimensional (but not necessarily irreducible) representation of $P^{\prime}$. Then there is an associated smooth homogeneous vector bundle $\mathscr{E}$ over $X$ with fiber $E$ at the identity coset $e P^{\prime}$. Similarly, $\mathscr{E}^{*}$ is the smooth homogeneous vector bundle over $X$ associated to the contragradient module $E^{*}$. In general, let $\Gamma_{X}(\mathscr{A})$ denote the smooth global sections of the smooth vector bundle $\mathscr{A}$ over $X$.

Since $X=G / P^{\prime}=K /\left(K \cap P^{\prime}\right)$ as homogeneous spaces, there is a unique $K$-invariant volume form $d \mu_{x}$ on $X$ with total volume 1 . We fix this volume form on $X$. Each $g \in G$ determines a diffeomorphism of $X$ via left multiplication and we obtain a corresponding modular function $c(g, x)$ defined by

$$
g^{*} d \mu_{x}=c(g, x) d u_{x}, \quad g \in G, x \in X .
$$

Following [W], we can now define a smooth $G$-action $\pi_{E}$ on $\Gamma_{X}(\mathscr{E})$ by

$$
\begin{aligned}
& {\left[\pi_{E}(g) f\right](x)=\left|c\left(g^{-1}, x\right)\right|^{1 / 2} g f\left(g^{-1} x\right),} \\
& \quad g \in G, x \in X, f \in \Gamma_{X}(\mathscr{E}) .
\end{aligned}
$$

Suppose $E$ and $F$ are two finite dimensional $P^{\prime}$ modules with associated $G$-modules $\Gamma_{X}(\mathscr{E})$ and $\Gamma_{X}(\mathscr{F})$ respectively. We denote by $\operatorname{Hom}_{G}\left(\Gamma_{X}(\mathscr{E}), \Gamma_{X}(\mathscr{F})\right)$ the space of continuous maps $L: \Gamma_{X}(\mathscr{E}) \rightarrow$ $\Gamma_{X}(\mathscr{F})$ which intertwine $\pi_{E}$ and $\pi_{F}$. The space of homogeneous differential operators is then

$$
\begin{aligned}
\mathbb{D}\left(\Gamma_{X}(\mathscr{E}), \Gamma_{X}(\mathscr{F})\right)= & \left\{D \in \operatorname{Hom}_{G}\left(\Gamma_{X}(\mathscr{E}), \Gamma_{X}(\mathscr{F})\right)\right. \\
& \left.\mid \operatorname{supp} D \phi \subseteq \operatorname{supp} \phi \text { for all } \phi \in \Gamma_{X}(\mathscr{E})\right\},
\end{aligned}
$$

where supp $\phi$ is the support of the section $\phi$.

For a finite dimensional $P^{\prime}$-module $E$, let $E$ also denote the $\mathfrak{p}$ module obtained by differentiation. Observe that the induced $\mathfrak{g}$-module $U_{\mathfrak{p}}\left(E^{*}\right)$ carries a compatible action of the finite group $S$. We can now state the desired lemma.

LEMMA 2.4. Let $E$ and $F$ be finite dimensional representations of $P^{\prime}$. Then there is a vector space isomorphism

$$
\mathbb{D}\left(\Gamma_{X}(\mathscr{E}), \Gamma_{X}(\mathscr{F})\right) \cong \operatorname{Hom}_{\mathfrak{g}, S}\left(U_{\mathfrak{p}}\left(F^{*}\right), U_{\mathfrak{p}}\left(E^{*}\right)\right) .
$$

Proof. Let $\mathscr{D}^{\prime}(\mathscr{E})=\mathscr{D}^{\prime}(X, \mathscr{E})$ denote the continuous dual of $\Gamma_{X}\left(\mathscr{E}^{*}\right)$ (where $\Gamma_{X}\left(\mathscr{E}^{*}\right)$ is given the usual Frechet topology, [H]), 
i.e. distributions, and denote by $\mathscr{D}_{0}^{\prime}(\mathscr{E})=\mathscr{D}_{0}^{\prime}(X, \mathscr{E})$ the subspace of $\mathscr{D}^{\prime}(\mathscr{E})$ consisting of distributions supported at the identity coset.

The exponential map realizes $\mathfrak{n}_{0}^{-}=\theta\left(\mathfrak{n}_{0}\right)$ as a coordinate patch around the identity coset $e P^{\prime}$ in $X$. Since $\mathfrak{n}_{0}^{-} \cong \mathbb{R}^{n}$, the vector valued version of Schwartz's theorem then gives a vector space isomorphism $T: U\left(\mathfrak{n}^{-}\right) \otimes E \rightarrow \mathscr{D}_{0}^{\prime}(\mathscr{E})$. We may describe this isomorphism explicitly as follows. We identity $\Gamma_{X}\left(\mathscr{E}^{*}\right)$ as a $G$-representation with the space $\mathscr{V}\left(\mathscr{E}^{*}\right)$ of functions on $G$ given by

$$
\begin{aligned}
\Gamma_{X}\left(\mathscr{E}^{*}\right) \cong \mathscr{V}\left(\mathscr{E}^{*}\right) & \\
=\left\{f: G \rightarrow E^{*} \mid f \text { is smooth, } f(g p)=\right. & \sigma_{E^{*}}(p)^{-1} f(g) \\
& \text { for } \left.g \in G, p \in P^{\prime}\right\},
\end{aligned}
$$

under the left regular representation on $\mathscr{V}\left(\mathscr{C}^{*}\right)$. Then the isomorphism of Schwartz's theorem is given by

$$
T(Y \otimes v)(\phi)=\left\langle R_{Y} \phi(e), v\right\rangle, \quad \phi \in \mathscr{V}, Y \in U\left(\mathfrak{n}^{-}\right), v \in E .
$$

Here $R_{Y}$ denotes right differentiation by $Y$ and $\langle$,$\rangle is the natural$ pairing on $E$ and $E^{*}$. Since $U_{\mathfrak{p}}(E) \cong U\left(\mathfrak{n}^{-}\right) \otimes E$, as vector spaces, a moderately tedious calculation shows that $T$, with the formula given above, becomes a well-defined $\mathfrak{g}$-module isomorphism

$$
T: U_{\mathfrak{p}}(E) \stackrel{\cong}{\rightarrow} \mathscr{D}_{0}^{\prime}(\mathscr{E}) \text {. }
$$

It now suffices to give an isomorphism

$$
\Psi: \mathbb{D}\left(\Gamma_{X}(\mathscr{E}), \Gamma_{X}(\mathscr{F})\right) \rightarrow \operatorname{Hom}_{\mathfrak{g}, S}\left(\mathscr{D}_{0}^{\prime}\left(\mathscr{F}^{*}\right), \mathscr{D}_{0}^{\prime}\left(\mathscr{E}^{*}\right)\right) .
$$

For $D \in \mathbb{D}\left(\Gamma_{X}(\mathscr{E}), \Gamma_{X}(\mathscr{F})\right)$, define $\Psi(D) \in \operatorname{Hom}_{\mathbb{C}}\left(\mathscr{D}^{\prime}\left(\mathscr{F}^{*}\right), \mathscr{D}^{\prime}\left(\mathscr{E}^{*}\right)\right)$ by $\Psi(D)(\mu)(\phi)=\mu(D \phi)$ for $\phi \in \Gamma_{X}(\mathscr{E})$ and $\mu \in \mathscr{D}^{\prime}\left(\mathscr{F}^{*}\right)$. The support condition on $D$ assures that $\Psi(D)$ preserves distributions supported at the identity coset. Also, for $Y \in \mathfrak{g}, \phi \in \Gamma_{X}(\mathscr{E})$ and $\mu \in \mathscr{D}^{\prime}\left(\mathscr{F}^{*}\right)$,

$$
\begin{aligned}
\Psi(D)(Y \mu)(\phi) & =Y \mu(D \phi)=\mu\left(d \pi_{F}(-Y) D \phi\right) \\
& =\mu\left(D\left(d \pi_{E}(-Y) \phi\right)\right)=(Y(\Psi(D) \mu))(\phi) .
\end{aligned}
$$

Thus $\Psi(D)$ commutes with the action of $\mathfrak{g}$. A similar calculation shows that $\Psi(D)$ commutes with $S$ and so we have a well-defined map $\Psi$ for (2.6). In order to show that $\Psi$ is an isomorphism we will construct an inverse map.

Fix a basis $v_{1}, \ldots, v_{t}$ for $F$ and let $v_{1}^{*}, \ldots, v_{t}^{*}$ be the dual basis of $F^{*}$. Identify $v_{i}^{*}$ with $1 \otimes v_{i}^{*}$ in $U_{\mathfrak{p}}\left(F^{*}\right)$. Let

$$
\Lambda \in \operatorname{Hom}_{\mathfrak{g}, S}\left(\mathscr{D}_{0}^{\prime}\left(\mathscr{F}^{*}\right), \mathscr{D}_{0}^{\prime}\left(\mathscr{E}^{*}\right)\right)
$$


and define

$$
\Phi: \operatorname{Hom}_{\mathfrak{g}, S}\left(\mathscr{D}_{0}^{\prime}\left(\mathscr{F}^{*}\right), \mathscr{D}_{0}^{\prime}\left(\mathscr{E}^{*}\right)\right) \rightarrow \operatorname{Hom}_{\mathbb{C}}\left(\Gamma_{X}(\mathscr{E}), \Gamma_{X}(\mathscr{F})\right)
$$

by the formula

$$
\Phi(\Lambda)(\phi)(x)=\sum_{i=1}^{t} \Lambda\left(T\left(1 \otimes v_{i}^{*}\right)\right)\left(\pi_{E}\left(x^{-1}\right) \phi\right) v_{i}, \quad x \in G, \phi \in \mathscr{V}(\mathscr{E}) .
$$

Notice that by linearity, $\Phi$ is independent of the basis chosen for $F$. Since $\Phi(\Lambda)$ can be computed in terms of differentiation by elements of $U\left(\mathfrak{n}^{-}\right)$translated to $x \in G$, it is clear that $\operatorname{supp}(\Phi(\Lambda)(\phi)) \subseteq$ $\operatorname{supp}(\phi)$. Thus $\Phi(\Lambda)$ is a differential operator. Moreover, for $g \in G$, since $\pi_{E}\left(x^{-1}\right) \pi_{E}(g)=\pi_{E}\left(\left(g^{-1} x\right)^{-1}\right)$, we see $\Phi(\Lambda)\left(\pi_{E}(g) \phi\right)(x)=$ $\Phi(\Lambda)(\phi)\left(g^{-1} x\right)=\pi_{F}(g) \Phi(\Lambda)(\phi)(x)$. Thus $\Phi(\Lambda)$ is $G$-homogeneous. So we have

$$
\Phi: \operatorname{Hom}_{\mathfrak{g}, S}\left(\mathscr{D}_{0}^{\prime}\left(\mathscr{F}^{*}\right), \mathscr{D}_{0}^{\prime}\left(\mathscr{E}^{*}\right)\right) \rightarrow \mathbb{D}\left(\Gamma_{X}(\mathscr{E}), \Gamma_{X}(\mathscr{F})\right) \text {. }
$$

Finally, for $\phi \in \mathscr{V}(\mathscr{E}), D \in \mathbb{D}\left(\Gamma_{X}(\mathscr{E}), \Gamma_{X}(\mathscr{F})\right)$ and $x \in G$ we have:

$$
\begin{aligned}
\Phi(\Psi(D))(\phi)(x) & =\sum T\left(1 \otimes v_{i}^{*}\right)\left(D \pi_{E}\left(x^{-1}\right) \phi\right) v_{i}=\left(D \pi_{E}\left(x^{-1}\right) \phi\right)(e) \\
& =\pi_{F}\left(x^{-1}\right) D \phi(e)=D \phi(x) .
\end{aligned}
$$

Thus $\Phi \circ \Psi$ is the identity. A similar computation shows that $\Psi \circ \Phi$ is the identity. This proves the isomorphism in (2.6) and completes the proof of (2.4).

3. Application to highest weight modules. In this section we apply (2.4) using the pair $\left(G, P^{0}\right)$, that is, we ignore the finite group $S$. We emphasize that the pair $\left(G, P^{0}\right)$ is not unique to the data $(\mathfrak{g}, \mathfrak{p})$.

Let $\mathscr{Z}(\mathfrak{g})$ denote the center of $U(\mathfrak{g})$. If $\theta$ is a character of $\mathscr{Z}(\mathfrak{g})$ and $M$ is an object in $\mathscr{O}_{\mathfrak{p}}$ let $M_{\theta}$ be the $U(\mathfrak{g})$-invariant subspace of $M$ on which $\mathscr{Z}(\mathfrak{g})$ acts by generalized character $\theta$. Then $M=$ $\bigoplus M_{\theta}$, and although the sum is taken over all characters $\theta$ it is a finite direct sum.

LEMMA 3.1. Let $E$ and $F$ be finite dimensional $\mathfrak{p}$-modules and $\theta$ a character of $\mathscr{Z}(\mathfrak{g})$. Then

$$
\operatorname{Hom}_{\mathfrak{g}}\left(U_{\mathfrak{p}}(E)_{\theta}, U_{\mathfrak{p}}(F)_{\theta}\right) \cong \operatorname{Hom}_{\mathfrak{g}}\left(U_{\mathfrak{p}}\left(F^{*}\right)_{\theta^{*}}, U_{\mathfrak{g}}\left(E^{*}\right)_{\theta^{*}}\right) .
$$

Proof. We begin by recalling that the function $c$ satisfies the cocycle relation

$$
c(g h, x)=c(g, h x) c(h, x), \quad g, h \in G, x \in X .
$$


Writing $e=g^{-1} g$ this formula leads us to the formula

$$
|c(g, x)|\left|c\left(g^{-1}, g x\right)\right|^{1 / 2}=|c(g, x)|^{1 / 2} .
$$

Define $A: \mathbb{D}\left(\Gamma_{X}(\mathscr{E}), \Gamma_{X}(\mathscr{F})\right) \rightarrow \mathbb{D}\left(\Gamma_{X}\left(\mathscr{F}^{*}\right), \Gamma_{X}\left(\mathscr{E}^{*}\right)\right)$ by taking the transpose adjoint with respect to $d \mu_{X}$, as in [H]. That is, for $D \in$ $\mathbb{D}\left(\Gamma_{X}(\mathscr{E}), \Gamma_{X}(\mathscr{F})\right), A(D)$ is defined by

$$
\int_{X}\langle\zeta, A(D) \phi\rangle d \mu_{x}=\int_{X}\langle D \zeta, \phi\rangle d \mu_{x}, \quad \zeta \in \Gamma_{X}(\mathscr{E}), \phi \in \Gamma_{X}\left(\mathscr{F}^{*}\right) .
$$

Let $g$ be in $G$. Using equation (3.2) we have for all $\zeta \in \Gamma_{X}(\mathscr{E})$ and $\phi \in \Gamma_{X}\left(\mathscr{E}^{*}\right)$ :

$$
\begin{aligned}
\int_{X}\left\langle\zeta, \pi_{E^{*}}(g) \phi\right\rangle d \mu_{x} & =\int_{X}\left\langle\zeta(x),\left|c\left(g^{-1}, x\right)\right|^{1 / 2} g \phi\left(g^{-1} x\right)\right\rangle d \mu_{x} \\
& =\int_{X}\left\langle\zeta(g x),\left|c\left(g^{-1}, g x\right)\right|^{1 / 2} g \phi(x)\right\rangle|c(g, x)| d \mu_{x} \\
& =\int_{X}\left\langle|c(g, x)|^{1 / 2} g^{-1} \zeta(g x), \phi(x)\right\rangle d \mu_{x} \\
& =\int_{X}\left\langle\pi_{E}\left(g^{-1}\right) \zeta(x), \phi(x)\right\rangle d \mu_{x} .
\end{aligned}
$$

Of course a similar equation holds if $\zeta$ is in $\Gamma_{X}(\mathscr{F})$ and $\phi$ is in $\Gamma_{X}\left(\mathscr{F}^{*}\right)$. It follows immediately that $A(D)$ is $G$-homogeneous whenever $D$ is $G$-homogeneous. Then $A$ is easily seen to be an isomorphism. In view of (2.4), this proves the lemma with the $\theta$ 's erased. The lemma then follows easily by tracing the action of $\mathscr{Z}(\mathfrak{g})$ as it is transformed under each of the specific isomorphisms used in the proof of (2.4) and under the action of $A$. This completes (3.1).

LEMMA 3.3. Let $E$ be in $\mathscr{A}_{\mathfrak{p}}$ and $\theta$ a character of $\mathscr{Z}(\mathfrak{g})$. Then there is a $\mathfrak{p}$-module $A$ in $\mathscr{A}_{\mathfrak{p}}$ and a $\mathfrak{p}$-module surjection $A \rightarrow E$ such that $U_{\mathfrak{p}}(A)_{\theta}$ is projective in $\mathscr{O}_{\mathfrak{p}}$.

Proof. This is implicit in 4.1 and 4.2 of [RC].

We can now complete the proof of Theorem 1.1 .

Proof of Theorem 1.1. Fix $E, F$ and $\theta$ as in Lemma 3.1. Write $\operatorname{Ext}_{\theta}^{k}\left(U_{\mathfrak{p}}(E), U_{\mathfrak{p}}(F)\right)$ for $\operatorname{Ext}_{\mathscr{O}_{\mathfrak{p}}}^{k}\left(U_{\mathfrak{p}}(E)_{\theta}, U_{\mathfrak{p}}(F)_{\theta}\right)$. Then we are to prove

$$
\begin{aligned}
\operatorname{dim} & \operatorname{Ext}_{\theta}^{k}\left(U_{\mathfrak{p}}(E), U_{\mathfrak{p}}(F)\right) \\
& =\operatorname{dim} \operatorname{Ext}_{\theta^{*}}^{k}\left(U_{\mathfrak{p}}\left(F^{*}\right), U_{\mathfrak{p}}\left(E^{*}\right)\right), \quad k \geq 0 .
\end{aligned}
$$


We proceed by induction on $k$, the case $k=0$ being (3.1). Choose $A \rightarrow E$ as in Lemma 3.3. Let $K=\operatorname{ker}(A \rightarrow E)$ so that we have a short exact sequence $0 \rightarrow K \rightarrow A \rightarrow E \rightarrow 0$. Then by the projectivity of $U_{\mathfrak{p}}(A)_{\theta}$ we have $\operatorname{Ext}_{\theta}^{1}\left(U_{\mathfrak{p}}(A), U_{\mathfrak{p}}(F)\right)=0$. There are two long exact sequences obtained by applying $\operatorname{Ext}_{\theta}^{*}\left(U_{\mathfrak{p}}(\cdot), U_{\mathfrak{p}}(F)\right)$ and $\operatorname{Ext}_{\theta^{*}}^{*}\left(U_{\mathfrak{p}}\left(F^{*}\right), U_{\mathfrak{p}}\left(\cdot^{*}\right)\right)$ to the short exact sequence $K \rightarrow A \rightarrow E$. Comparing the first 5 terms of these sequences and using (3.1) we see:

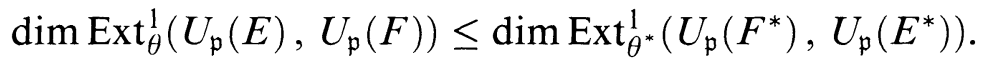

However, this argument is valid for the pair $\left(F^{*}, E^{*}\right)$ as well as for the pair $(E, F)$. So we see that the inequality in $(3.5)$ is actually an equality, establishing (3.4) for $k=1$.

For $k>1$ the projectivity of $U_{\mathfrak{p}}(A)_{\theta}$ and comparison of the two long exact sequences gives (using induction)

$$
\text { (3.6) } \begin{aligned}
\operatorname{dim} \operatorname{Ext}_{\theta}^{k}\left(U_{\mathfrak{p}}(E), U_{\mathfrak{p}}(F)\right) & =\operatorname{dim} \operatorname{Ext}_{\theta}^{k-1}\left(U_{\mathfrak{p}}(K), U_{\mathfrak{p}}(F)\right) \\
& =\operatorname{dim} \operatorname{Ext}_{\theta^{*}}^{k-1}\left(U_{\mathfrak{p}}\left(F^{*}\right), U_{\mathfrak{p}}\left(K^{*}\right)\right) \\
& \leq \operatorname{dim} \operatorname{Ext}_{\theta^{*}}^{k}\left(U_{\mathfrak{p}}\left(F^{*}\right), U_{\mathfrak{p}}\left(E^{*}\right)\right)
\end{aligned}
$$

since by induction $\operatorname{Ext}_{\theta^{*}}^{k-1}\left(U_{\mathfrak{p}}\left(F^{*}\right), U_{\mathfrak{p}}\left(A^{*}\right)\right)=\operatorname{Ext}_{\theta}^{k-1}\left(U_{\mathfrak{p}}(A), U_{\mathfrak{p}}(F)\right)$ $=0$. Again by symmetry in the argument, the inequality in (3.6) must be an equality. This establishes (3.4) and proves Theorem 1.1.

4. Application to real groups. Consider now the case when $P=P^{\prime}$; then $P$ is not necessarily connected. As before put $X=G / P$. Given a finite dimensional $P$-module $E$ we let $I_{P}(E)$ denote the space of $K$-finite vectors in $\Gamma_{X}(\mathscr{E})$. If $E$ is irreducible and trivial as an $N$ module we refer to these as degenerate series representations of $G$. In case $P=P_{m}$, these are usually referred to as principal series representations. A general problem in the subject is to parameterize the intertwining operators between any two degenerate series representations. Since the $K$-finite vectors of $\Gamma_{X}(\mathscr{E})$ are dense in $\Gamma_{X}(\mathscr{E})$, we have an inclusion (via the restriction map)

$$
\mathbb{D}\left(\Gamma_{X}(\mathscr{E}), \Gamma_{X}(\mathscr{F})\right) \hookrightarrow \operatorname{Hom}_{\mathfrak{g}, K}\left(I_{P}(E), I_{P}(F)\right) .
$$

Denote by $\operatorname{Diff}\left(I_{P}(E), I_{P}(F)\right)$ the image of the map in (4.1). These are the differential intertwining operators. We may apply (2.4) and conclude that the differential intertwining operators correspond to $\operatorname{Hom}_{\mathfrak{g}, S}\left(U_{\mathfrak{p}}\left(F^{*}\right), U_{\mathfrak{p}}\left(E^{*}\right)\right)$, where $S$ is $(\mathbb{Z} / 2 \mathbb{Z})^{m}$ for some $m$. 
As an example, let $G$ be the real Lie group $\operatorname{SL}(2, \mathbb{R})$ and consider the category $\mathscr{H C}_{F}$ of admissible representations of $G$ having the same infinitesimal character as a fixed irreducible finite dimensional $G$-representation $F$. Then $\mathscr{H C}_{F}$ contains two reducible principal series representations which we denote by $I_{P}$ (quo) and $I_{P}(\mathrm{sub})$, indicating that $F$ is the unique irreducible quotient or subrepresentation respectively. As is well known,

$$
\operatorname{dim}_{\mathbb{C}} \operatorname{Hom}_{\mathfrak{g}, K}\left(I_{P}(\text { quo }), I_{P}(\mathrm{sub})\right)=1
$$

and

$$
\operatorname{dim}_{\mathbb{C}} \operatorname{Hom}_{\mathfrak{g}, K}\left(I_{P}(\mathrm{sub}), I_{P}(\text { quo })\right)=2 .
$$

Using (2.4) we thus obtain

$$
\operatorname{dim}_{\mathbb{C}} \operatorname{Diff}_{\mathfrak{g}, K}\left(I_{P}(\mathrm{sub}), I_{P}(\text { quo })\right)=1
$$

and

$$
\operatorname{dim}_{\mathbb{C}} \operatorname{Diff}_{\mathfrak{g}, K}\left(I_{P}(\text { quo }), I_{P}(\mathrm{sub})\right)=0 .
$$

Similar calculations may be carried out in the case of any real rank one Lie group. This follows from [C1] and [C2].

5. The extension problem. We were initially led to Theorem 1.1 through an interest in the Extension Problem for the category $\mathscr{O}_{\mathfrak{p}}$. To see the connection with our theorem let $\mathscr{O}_{0}$ be the subcategory of $\mathscr{O}_{\mathfrak{p}}$ consisting of modules with the same generalized infinitesimal character as the trivial $\mathfrak{g}$-module. Denote by $\mathscr{W}$ (respectively $\mathscr{W}_{\mathfrak{l}}$ ) the Weyl group of $\mathfrak{g}$ (respectively $\mathfrak{l}$ ) and recall that each coset of $\mathscr{W}_{\mathfrak{l}} \backslash \mathscr{W}$ admits a unique coset representative of minimal length. Denote by $\mathscr{W}^{l}$ the collection of these representatives. Let $w_{0}$ (resp. $w_{l}$ ) be the longest element of $\mathscr{W}$ (respectively $\mathscr{W}_{l}$ ). Let $\rho$ be the half sum of the positive weights of $\mathfrak{h}$ in $\mathfrak{g}$. Then for each $x \in \mathscr{W}^{\mathfrak{l}}$ we denote by $E_{x}$ the irreducible l-module of highest weight $w_{l} x w_{0} \rho-\rho$ and consider $E_{x}$ as a $\mathfrak{p}$-module by letting $\mathfrak{n}$ act trivially. Set $V_{x}=U_{\mathfrak{p}}\left(E_{x}\right)$. These are the generalized Verma modules in $\mathscr{O}_{0}$. We denote by $L_{x}$ the unique irreducible quotient of $V_{x}$. Finally, for each $y, w \in \mathscr{W}^{l}$, define polynomials

$$
E_{y, w}(q)=\sum_{k \geq 0}(-1)^{k} q^{k} \operatorname{dim}_{\mathbb{C}} \operatorname{Ext}_{\mathscr{O}_{p}}^{k}\left(V_{y}, V_{w}\right)
$$

The Extension Problem is just the problem of computing in some fashion the polynomials $E_{y, w}(q)$. In general, this problem is as yet 
unsolved and there are not even conjectures for the form of the answer. However, if $\mathfrak{p}$ is a Borel subalgebra then there is a conjectured recursion formula for the polynomials, cf. [GJ], and if $\mathfrak{g}$ and $\mathfrak{p}$ form an indecomposable Hermitian symmetric pair then there is a known recursion formula, $\mathrm{cf}$. [S]. Theorem 1.1 imposes an interesting relation on these generating functions. To state this carefully, recall that there exists an order reversing involution $d: \mathscr{W}^{l} \rightarrow \mathscr{W}^{l} ; d(x)=w_{l} x w_{0}$. As a corollary to (1.1) we obtain:

COROLlary 5.2. For all $y, w \in \mathscr{W}^{l}$,

$$
E_{y, w}=E_{d(w), d(y)} \text {. }
$$

In low rank examples, (5.2) can be an effective tool in solving the extension problem. For the remainder of this section we will sketch a representative example of such a computation.

Let $\mathfrak{g}$ be $\mathfrak{s l}_{4}(\mathbb{C})$ and denote the simple roots of $\mathfrak{g}$ by $\alpha_{1}, \alpha_{2}$, and $\alpha_{3}$. Choose $\mathfrak{p}$ to be the maximal parabolic subalgebra whose Levi factor $\mathfrak{l}$ has simple roots $\alpha_{1}$ and $\alpha_{3}$. If $s_{i}$ denotes the simple reflection corresponding to $\alpha_{i}$, then

$$
\mathscr{W}^{\mathfrak{l}}=\left\{e, s_{2}, s_{2} s_{3}, s_{2} s_{1}, s_{2} s_{3} s_{1}, s_{2} s_{3} s_{1} s_{2}\right\} .
$$

In this simple case, the spaces $\operatorname{Ext}_{\mathscr{O}_{\mathrm{p}}}^{k}\left(V_{y}, L_{w}\right)$ are known explicitly for all $y$ and $w$ in $\mathscr{W}^{l}$. Furthermore there are explicit formulas for the (unique) Loewy series of the modules $V_{w}$. All of this information can be found in either [CC] or [ES]. By combining these two pieces of information and analyzing the collection of long exact sequences which they give rise to, one can easily compute all of the spaces $\operatorname{Ext}_{\mathscr{O}_{\mathrm{p}}}^{k}\left(V_{y}, V_{w}\right)$ except for the single case $y=e, w=s_{2} s_{3} s_{1}$ and $k=1$. This last case is handled by Theorem 1.1. Since $d(e)=s_{2} s_{3} s_{1} s_{2}$ and $d\left(s_{2} s_{3} s_{1}\right)=s_{2}$ we have

$$
\operatorname{dim}_{\mathbb{C}} \operatorname{Ext}_{\mathscr{O}_{p}}^{1}\left(V_{e}, V_{s_{2} s_{3} s_{1}}\right)=\operatorname{dim}_{\mathbb{C}} \operatorname{Ext}_{\mathscr{O}_{p}}^{1}\left(V_{s_{2}}, V_{s_{2} s_{3} s_{1} s_{2}}\right) .
$$

The right-hand side of (5.3) is easily shown (by the methods mentioned above) to be zero. This allows one to compute the following table for the polynomials $E_{y, w}(q)$.

REMARK. We must point out that in this simple example the polynomials $E_{y, w}(q)$ can also be computed by the recursion formulas given in [S]. 
TABLE 1

Generating polynomials $E_{y, w}$ for $\mathscr{O}_{0}$

\begin{tabular}{|l|l|l|l|l|l|l|}
\hline$y w$ & $e$ & $s_{2}$ & $s_{2} s_{3}$ & $s_{2} s_{1}$ & $s_{2} s_{3} s_{1}$ & $s_{2} s_{3} s_{1} s_{2}$ \\
\hline$e$ & 1 & $1-q$ & $-q+q^{2}$ & $-q+q^{2}$ & $q^{2}-q^{3}$ & $1-q-q^{3}+q^{4}$ \\
\hline$s_{2}$ & 0 & 1 & $1-q$ & $1-q$ & $1-2 q+q^{2}$ & $q^{2}-q^{3}$ \\
\hline$s_{2} s_{3}$ & 0 & 0 & 1 & 0 & $1-q$ & $-q+q^{2}$ \\
\hline$s_{2} s_{1}$ & 0 & 0 & 0 & 1 & $1-q$ & $-q+q^{2}$ \\
\hline$s_{2} s_{3} s_{1}$ & 0 & 0 & 0 & 0 & 1 & $1-q$ \\
\hline$s_{2} s_{3} s_{1} s_{2}$ & 0 & 0 & 0 & 0 & 0 & 1 \\
\hline
\end{tabular}

\section{REFERENCES}

[BGG] I. N. Bernstein, I. M. Gelfand and S. I. Gelfand, Differential operators on the base affine space and a study of $\mathfrak{g}$-modules in Lie Groups and their representations, I. M. Gelfand editor, 21-64, Halsted Press, 1975.

[B] B. Boe, Homomorphisms between generalized Verma modules, $\mathrm{Ph}$. D. Thesis, Yale University, 1982.

[CC] L. Casian and D. Collingwood, The Kazhdan Lusztig conjecture for generalized Verma modules, Math. Z., 195 (1987), 581-600.

[C1] D. Collingwood, Representations of rank one Lie groups, Research Notes in Mathematics 137, Pitman Publishing, 1985.

[C2] __ Representations of rank one Lie groups II: $\mathfrak{n}$-cohomology, Memoirs of the Amer. Math. Soc., 387 (1988).

[D] J. Dixmier, Enveloping Algebras, North-Holland Press, 1977.

[ER] M. Eastwood and J. Rice, Conformally invariant differential operators on Minkowski space, Comm. in Math. Phys., 109 (1987), 207-228.

[ES] T. J. Enright and B. Shelton, Categories of highest weight modules: applications to classical Hermitian symmetric pairs, Memoirs of the Amer. Math. Soc., 367 (1987).

[GJ] O. Gabber and A. Joseph, Towards the Kazhdan-Lusztig conjecture, Ann. Scient. Ec. Norm. Sup., 14 (1981), 261-302.

[H] S. Helgason, Groups and Geometric Analysis, Academic Press, 1984.

[J] H. Jakobsen, Basic covariant differential operators, Ann. Scient. Ec. Norm. Sup., 4 serie, t. 18, (1985), 421-436.

[KZ] A. Knapp and G. Zuckerman, Classification of irreducible tempered representations of semisimple groups, Annals of Math., 116 (1982), 389-501.

[L] J. Lepowsky, Conical vectors in induced modules, Trans. Amer. Math. Soc., 208 (1975), 218-272.

[RC] A. Rocha-Caridi, Splitting criteria for $\mathfrak{g}$-modules induced from a parabolic and the Bernstein-Gelfand-Gelfand resolution of a finite-dimensional, irreducible g-module, Trans. Amer. Math. Soc., 262 (1980), 335-366.

[S] B. Shelton, Extensions between generalized Verma modules: the Hermitian symmetric cases, Math. Z., 197 (1988), 305-318. 
[W] N. Wallach, Harmonic Analysis on Homogeneous Spaces, Marcel Dekker, 1973.

Received December 12, 1988 and in revised form July 11, 1989. The first author was supported in part by NSF Grant DMS- 8800384 .

UNIVERSITY OF WASHINGTON

SeATtLe, WA 98195

AND

UNIVERSITY OF OREGON

EUGENE, OR 97403 



\section{PACIFIC JOURNAL OF MATHEMATICS EDITORS}

\author{
V. S. VARADARAJAN \\ (Managing Editor) \\ University of California \\ Los Angeles, CA 90024-1555-05 \\ Herbert Clemens \\ University of Utah \\ Salt Lake City, UT 84112 \\ Thomas EnRIght \\ University of California, San Diego \\ La Jolla, CA 92093
}

R. FINN

Stanford University

Stanford, CA 94305

HeRmanN FlaschKa

University of Arizona

Tucson, AZ 85721

VAughan F. R. Jones

University of California

Berkeley, CA 94720

Steven Kerckhoff

Stanford University

Stanford, CA 94305

\section{C. Moore}

University of California

Berkeley, CA 94720

Martin ScharlemanN

University of California

Santa Barbara, CA 93106

Harold Stark

University of California, San Diego

La Jolla, CA 92093

\section{ASSOCIATE EDITORS}
R. ARENS
E. F. BECKENBACH
B. H. NEUMANN
F. WoLF
K. YoshidA (1906-1982)
(1904-1989)

\section{SUPPORTING INSTITUTIONS}

UNIVERSITY OF ARIZONA
UNIVERSITY OF BRITISH COLUMBIA
CALIFORNIA INSTITUTE OF TECHNOLOGY
UNIVERSITY OF CALIFORNIA
MONTANA STATE UNIVERSITY
UNIVERSITY OF NEVADA, RENO
NEW MEXICO STATE UNIVERSITY
OREGON STATE UNIVERSITY

\author{
UNIVERSITY OF OREGON \\ UNIVERSITY OF SOUTHERN CALIFORNIA \\ STANFORD UNIVERSITY \\ UNIVERSITY OF HAWAII \\ UNIVERSITY OF TOKYO \\ UNIVERSITY OF UTAH \\ WASHINGTON STATE UNIVERSITY \\ UNIVERSITY OF WASHINGTON
}

The Supporting Institutions listed above contribute to the cost of publication of this Journal, but they are not owners or publishers and have no responsibility for its content or policies.

Mathematical papers intended for publication in the Pacific Journal of Mathematics should be in typed form or offset-reproduced (not dittoed), double spaced with large margins. Please do not use built up fractions in the text of the manuscript. However, you may use them in the displayed equations. Underline Greek letters in red, German in green, and script in blue. The first paragraph must be capable of being used separately as a synopsis of the entire paper. In particular it should contain no bibliographic references. Please propose a heading for the odd numbered pages of less than 35 characters. Manuscripts, in triplicate, may be sent to any one of the editors. Please classify according to the 1980 Mathematics Subject Classification (1985 Revision) scheme which can be found in the December index volumes of Mathematical Reviews. Supply name and address of author to whom proofs should be sent. All other communications should be addressed to the managing editor, or Elaine Barth, University of California, Los Angeles, California 90024-1555-05.

There are page-charges associated with articles appearing in the Pacific Journal of Mathematics. These charges are expected to be paid by the author's University, Government Agency or Company. If the author or authors do not have access to such Institutional support these charges are waived. Single authors will receive 50 free reprints; joint authors will receive a total of 100 free reprints. Additional copies may be obtained at cost in multiples of 50 .

The Pacific Journal of Mathematics (ISSN 0030-8730) is published monthly. Regular subscription rate: $\$ 190.00$ a year (12 issues). Special rate: $\$ 95.00$ a year to individual members of supporting institutions.

Subscriptions, orders for numbers issued in the last three calendar years, and changes of address should be sent to Pacific Journal of Mathematics, P.O. Box 969, Carmel Valley, CA 93924, U.S.A. Old back numbers obtainable from Kraus Periodicals Co., Route 100, Millwood, NY 10546.

The Pacific Journal of Mathematics at P.O. Box 969, Carmel Valley, CA 93924 (ISSN 0030-8730) is published monthly. Second-class postage paid at Carmel Valley, California 93924, and additional mailing offices. Postmaster: send address changes to Pacific Journal of Mathematics, P.O. Box 969, Carmel Valley, CA 93924.

PUBLISHED BY PACIFIC JOURNAL OF MATHEMATICS, A NON-PROFIT CORPORATION

Copyright (C) 1990 by Pacific Journal of Mathematics 


\section{Pacific Journal of Mathematics}

\section{Vol. 146, No. 2 December, 1990}

David Fenimore Anderson, David Earl Dobbs, Paul M. Eakin, Jr. and

William James Heinzer, On the generalized principal ideal theorem and Krull domains .................................... 201

Richard C. Bradley, On $\rho$-mixing except on small sets $\ldots \ldots \ldots \ldots \ldots 217$

David H. Collingwood and Brad Shelton, A duality theorem for extensions

of induced highest weight modules $\ldots \ldots \ldots \ldots \ldots \ldots \ldots \ldots \ldots \ldots 27$

Gerd H. Fricke and John Albert Fridy, Sequence transformations that guarantee a given rate of convergence $\ldots . \ldots \ldots \ldots \ldots \ldots \ldots . \ldots . \ldots . \ldots 239$

Michael Justin Heumos and Stephen James Rallis, Symplectic-Whittaker

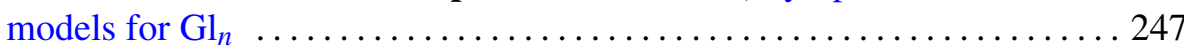

Severino Toscano do Rego Melo, A comparison algebra on a cylinder with

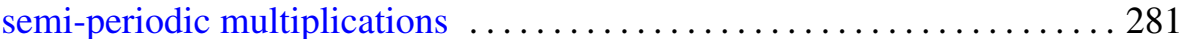

Daniel Pecker, On the elimination of algebraic inequalities ........... 305

Geoffrey Lynn Price, The $C^{*}$-algebras generated by pairs of semigroups of isometries satisfying certain commutation relations $\ldots \ldots \ldots \ldots \ldots \ldots 315$

John Kurt Sauter, Jr., Isomorphisms among monodromy groups and

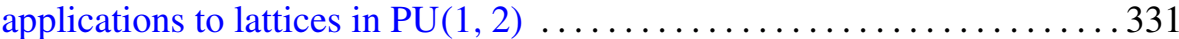

Shoji Yokura, A formula for Segre classes of singular projective varieties . . 385 